\title{
Spatial Accessibility of Road Network in Wuhan Metropolitan Area Based on Spatial Syntax
}

\author{
Chengliang Liu*, Ruilin Yu \\ School of Urban and Environmental Science, Huazhong Normal University, Wuhan, China \\ Email: *chenglianglew@163.com
}

Received December 8, 2011; revised January 18, 2012; accepted January 29, 2012

\begin{abstract}
Based on space syntax theory, the spatial accessibility of the road network in Wuhan Metropolitan Area has been quantitatively analyzed by building a series of accessibility variables. Topologic connectivity in the accessible rings appears to be broken; traffic axis network is in spatial structure of hub-and-spoke and fishbone-like. Meanwhile, the differences in classified road network have led to inefficiency of its network servo and its ever-worsening capability to respond to traffic jams. Besides, two band-like integrated cores of which one is east to west along the Yangtze River and the other is north to south along Beijing to Guangzhou Railway, have become the first level traffic axis in the whole network, which is responsible for the connectivity of the entire metropolitan area network. This consequently has strengthened the dominant position of Wuhan which is located on the bands' crossing. In short, the spatial accessibility of that classified space morphology, the urban system, the transport infrastructure as well as the social and economic development of Wuhan Metropolitan Area are highly interrelated to each other, especially to the high level highway network featured by freeways, the development level of which is well in line with that of road network accessibility.
\end{abstract}

Keywords: Accessibility; Road Network; Space Syntax; Wuhan Metropolitan Area

\section{Introduction}

Spatial accessibility has become a prerequisite to the integration of the urban center and its circumference [1]. The spatial evolution of metropolitan area and the development of its transport network are in interactive process [2]. A well-developed transport network has become the basic condition and essential prerequisite to the systematic operation of the whole metropolitan area, the accessibility of which determines whether or not the material flow, the energy flow as well as the information flow is smooth between the urban center and its circumference.

Through accessibility analysis, the interactive degree between the urban center and its circumference can be well reflected [3]. So are the exchange opportunities and potentiality in social, economic, cultural and technology sections between the two parts. And it is the focus of current researching field that revealing the geo-spatial characteristics of the metropolitan area and analyzing and evaluating the spatial structure of that by studying its transport network and spatial accessibility between the urban center and its circumference [4].

As to what to research, temporarily four propositions deserve our much more attention. Namely, how to define and assess accessibility, how to apply the results of ac-

${ }^{*}$ Corresponding author. cessibility assessment to the analysis of spatial structure and the upgrading of new transportation system and communication technology, and lastly how accessibility influences the metropolitan spatial morphology: 1) When it comes to the definition and assessment of accessibility, all agrees that accessibility is so flexible (slippery) a concept that different scholars have different comprehension of its connotation as accessibility refers to not only objectively how convenient the network transportation is, it also subjectively means the preference to which nodes to choose [5]. Also, accessibility refers to transport infrastructure and the morphological assessment of spatial units (railway, road, aviation, terminal ports, stations and integrated transport, etc.) and public service infrastructures (green parks, hospitals, shopping malls, schools, etc.) as well as the linkage of cyberspaces in communication systems [6]; 2) The analysis on spatial accessibility shows that the urban network accessibility and the construction of infrastructures in transport network as well as the central cities' level are highly interrelated. The metropolitan spatial morphology is in "hub-and-spoke" structure and is in the spatial pattern of concentric layered circles [7-10];3) The assessment on accessibility of the ever-upgraded new transportation system and communication technology is mainly concentrated on the effects building freeways has on the accessibility of the 
whole metropolitan region [11,12]; 4) As to the assessment on accessibility of metropolitan spatial morphology, it mainly focuses on the formation and development of urban transport network and urban corridors under the converging effect of transport network [13,14].

As to the researching methods, the econometric analysis on accessibility of transport network has been very popular recently. The main idea is to abstract the transport network into a geometric network or topological network and then evaluate the accessibility of it in the following four dimensions; namely, first, the spatial separation (such as distance measurement method mainly concerning with the actual distance, commuting time and the transportation costs); second, the spatial effects (such as gravitation measurement method represented by Hansen's Potential Model and Shen's Supply and Demand Model); third, the accumulation of opportunities (such as accumulation of Opportunity Act which bases evaluation on the exchanging opportunities of working, studying, shopping, health care, entertaining); and lastly, the spatial connectivity (such as topology measurement method, considering only the connectivity of the network and regardless of its actual distance) [15-18]. In recent years, due to the ever-increasing introduction of GIS spatial analysis method and graph theory [19], some scholars begin to use space syntax to analyze connective characteristics of the topological network [20-22].

In summary, quantitative evaluation of accessibility has now become popular. It mainly focuses on urban transportation and services as well as its location assessment. However, the research on the law of the metropolitan area's internal association and interaction is relatively weak. What's more, the studying scales are merely two levels - the nationwide (worldwide) $[23,24]$ and the circumference (within urban center) [25]. Worse still, the Empirical Study on the accessibility of the intercity road network in metropolitan region hasn't much been conducted $[26,27]$. In speaking of researching method, the disadvantage is that single measuring methods has much been carried out while multi-scale quantitative analysis on accessibility under econometric models is far from enough [28]. What's worse, the space syntax researches on topological network rarely see breakthroughs made. Almost all relevant researches are concentrated on inner cities [29]; the innovative researches on syntactical level on the structure of metropolitan road network are also far from enough.

Temporarily, our country is in the period of rapid development of urbanization [30]. As the metropolises are being grouped and integrated, there arises an increasingly obvious problem of the transport structure, a problem which can be properly addressed by optimizing the road network development pattern. Taking Wuhan Metropolitan Area as an example, this article aims at providing theoretical and empirical reference to Metropolitan Transportation Planning in China by evaluating the spatial law of metropolitan road network from different perspectives by analyzing a series of spatial syntactic variables built by GIS spatial analysis techniques.

\section{Study Area and Methods}

\subsection{The Scope of the Study}

Due to the distance attenuation principle, the Wuhan Metropolitan Area with Wuhan City as its centre and several other cities as its circumference has three levels: the core ring (including Wuhan, E'zhou, Huangshi, Xiaogan four cities, altogether forming a dense urban area named as Wu-E-Huang-Xiao urban compact district); the middle ring (Huanggang, Xiantao, Tianmen, Qianjiang, Xianning five cities); the external ring (Suizhou, Xinyang, Jiujiang, Yueyang, Jingzhou, Jingmen six cities) [31] (see Figure 1).

\subsection{Module Application}

Space Syntax module is in fact based on accessibility and integration of topological network, the fundamental idea of which is to firstly abstract the spaces into the connective map; secondly, to analyze the axis or the nodes by using topology method to lastly build a series of variables.

- Connectivity value $C_{i}$

In the connection diagram, the connectivity is the total number of any spatial unit connected to a given spatial unit $i$, which reflects its capability to be connected to other spatial units in the topological network. It can be expressed as follows:

$$
C_{i}=k
$$

where $k$ refers to the total number of spatial units directly connected to the given spatial unit.

- Control value $c t r l_{i}$

The control value means that how well the given spatial unit can control those spatial units connected to it, which reflects its control strength over and influences on any spatial unit connected. It can be expressed as follows:

$$
\operatorname{ctrl}_{i}=\sum_{j=1}^{k} \frac{1}{C_{i}}
$$

\section{- Depth value $D$}

The depth value is a minimum number of spatial units in certain step-distance which are connected to a given spatial unit. In the connection diagram, it reflects the shortest step-distance measured between a given spatial unit and any other spatial unit (not the real measured distance). Setting the shortest step-distance between the given spatial unit and any other spatial unit is $d$ ( $d$ is a 


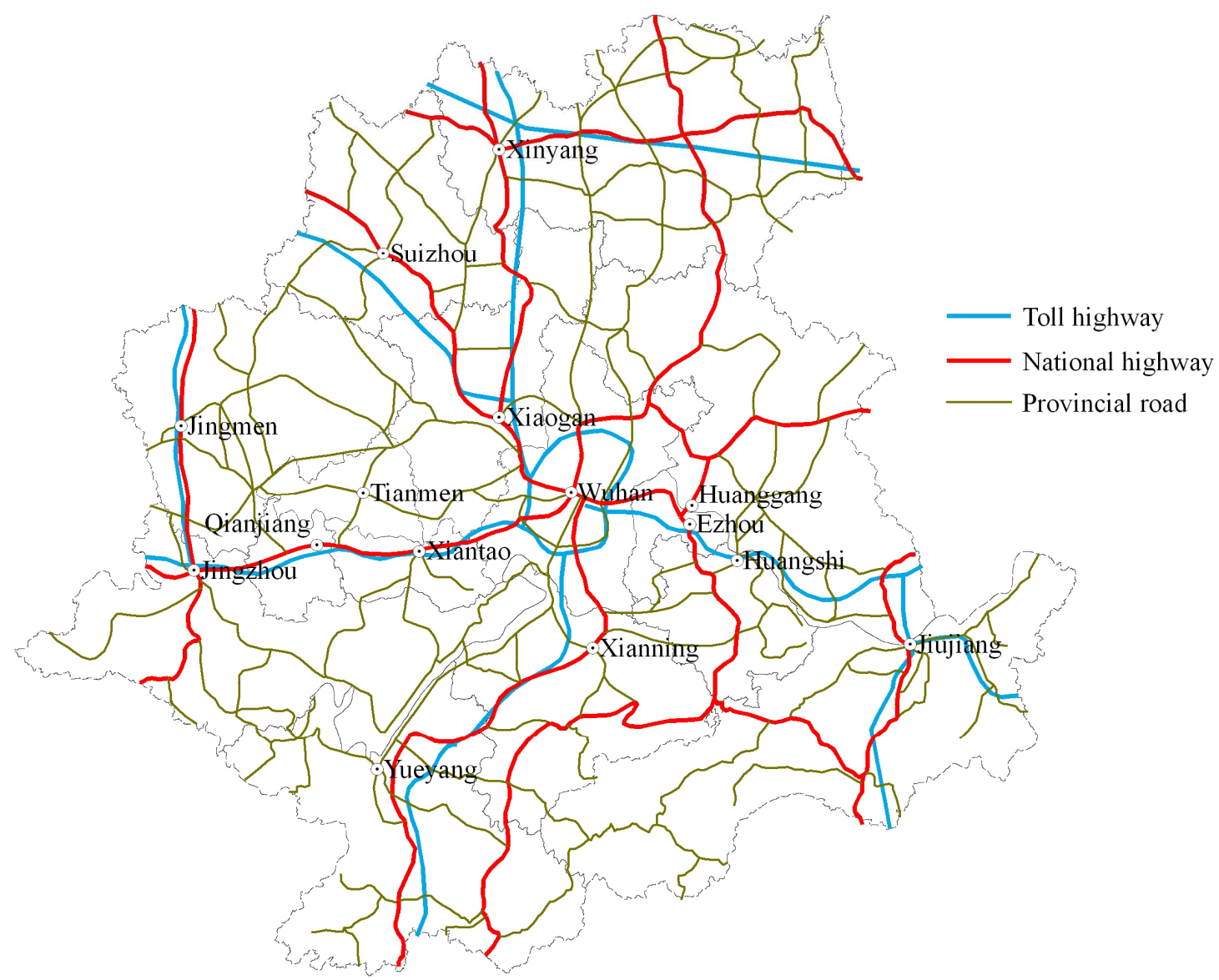

Figure 1. The high-grade transport network distribution.

integer), whose minimum number is 1 and maximum is $\mathrm{s}$ and the shortest step-distance number is $N_{d}$. $D_{i}$ can be expressed as follows:

$$
D=\sum_{d=1}^{s} d \times N_{d}=\left\{\begin{array}{lll}
\text { if } & d=1 & \text { then }=\text { connectivity } \\
\text { if } & d=h & \text { then }=\text { localdepth } \\
\text { if } & d=s & \text { then }=\text { globaldepth }
\end{array}\right.
$$

where the condition set is that $1<d<s$. When $d=1$, which reflects the total number of any spatial unit connected to the given unit, the depth value is one step-distance, namely, the connectivity value; when the value of $d$ is on steady increase, the depth value is also gradually increasing. In this case, the depth value is local depth value or $h$-step depth value; when $d=s$, the depth value is the global depth. In practice, the mean depth value $D_{i}$ is usually used, which can be expressed as follows:

$$
\bar{D}=\frac{\sum_{\mathrm{d}=1}^{s} \mathrm{~d} \times N_{\mathrm{d}}}{n-1}
$$

In formula (4), $n$ is the number of node to be studied, $n-1$ means that there are at most $n-1$ to-be-studied nodes connected to the given node.

- Integration value $R A_{i} / R R A_{i}$
The integration value reflects the degree of centralization or dispersion between a given spatial unit and all other spatial units in an urban system. It reflects how many steps it will need to visit all the other spatial units starting from the given one. The integration value can be expressed by relative asymmetry (briefly $R A_{i}$ ) and real relative asymmetry (briefly $R R A_{i}$ ). Generally speaking, when $R A_{i} / R R_{i}>1$, the spatial unit's degree of centralization is higher; when $0.4<R A_{i} / R R A_{i}<0.6$, the spatial units are relatively dispersed. Judging by the nodes' chosen scale, integration value can be classified into two categories: the local integration and the global integration. The global integration denotes the topological relation between a given spatial unit and any other spatial unit in an urban system, while the local one denotes the interactive relation between a given spatial unit and those spatial units several-step distance away from it (usually three-step distance). It can be expressed as follows:

$$
R A_{i}=\frac{2(\bar{D}-1)}{n-2} \quad R R A_{i}=\frac{R A_{i}}{D_{n}}
$$

where $n$ refers to the total number of axial lines or nodes in the system; $D$ stands for the mean depth value. And: 


$$
D_{n}=\frac{2\left\{n\left[\log _{2}((n+2) / 3)-1\right]+1\right\}}{(n-1)(n-2)}
$$

\subsection{Data Processing}

With the help of ArcGIS9.0 software, spatial database of the high-grade road network in Wuhan Metropolitan Area is established based on Transportation Atlas of China (2010) (Figure 1). By digitalizing the axis, thematic axial maps of the high-grade road network in Wuhan Metropolitan Area is attained as well. By checking the topological relations of the axial map where the isolated and suspended arcs' interference being excluded and by measuring the spatial syntactic variables, topological relations is expressed in icon (Figure 2) according to Equations (1)-(6) and under Axwoman module.

\section{Analysis on Spatial Accessibility Based on Space Syntax}

\subsection{The Overall Level of the Network Topological Connectivity Is Not So High that Its Servo Capacity Is Weakened and the Effectiveness of the Whole Network in Not Maximized to the Fullest}

Connectivity, control value and depth value as well as other indicators all reflect the connectivity and the im- portance of the traffic axis to any spatial unit in a system. The mean connectivity value of the entire transport network of Wuhan Metropolitan Area is merely 4.54, and the mean control value merely 1.10 . Meanwhile, the traffic axis whose connectivity is less than 8 accounts for $94.25 \%$ of the total amount of axis while whose control value is less than $2.0085 .62 \%$; whereas the traffic axis with more control strength and higher connectivity (connectivity $\geq 9$, the control value $\geq 2$, the depth value $\geq 50$ ) is in minority and is relatively densely distributed. As a result, the spatial road network of the whole Wuhan Metropolitan Area is not well controlled and infiltrated and the overall level of the topological connectivity of the high-grade road network is relatively low. Therefore, the efficiency of road network is to some extend negatively influenced.

\subsection{Network Topological Connectivity Is Significantly Classified in the Pyramid-Like Structure; the Development of the Road Network Is Immature}

Each spatial syntactical variable of road network is significantly classified, forming three levels: The first level traffic axial lines' connectivity $(C)$ is $\geq 9$, the control value (ctrl) $\geq 2$, three-step depth value $(D) \geq 50$ and integrated transport axis $R A \geq 1.7$, accounting for no more

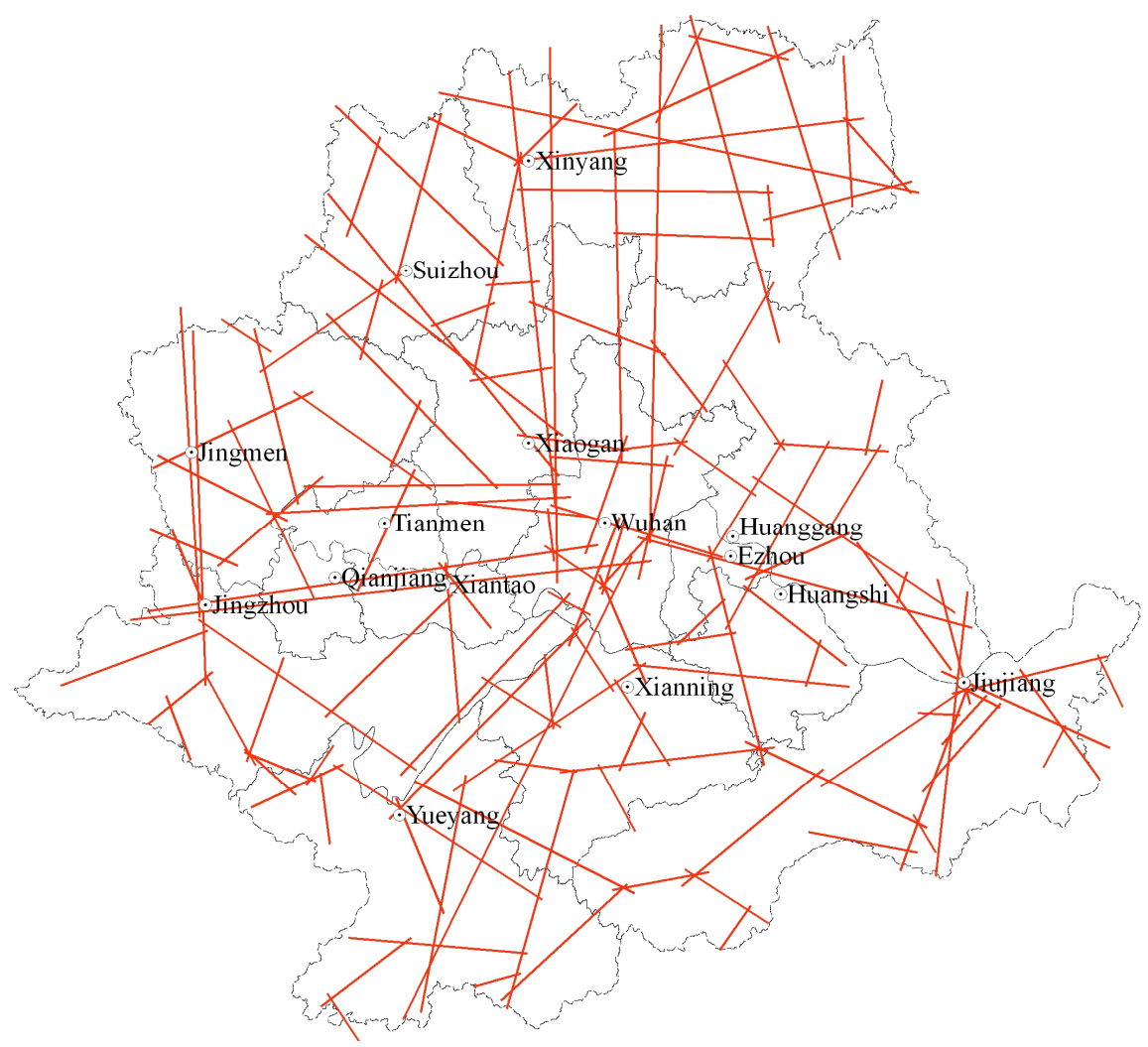

Figure 2. The axis map of road network of Wuhan metropolitan area. 
than $15 \%$ of all the transport axis, and of the $15 \%$ share, traffic axis with $C \geq 9$ and $D \geq 50$ takes up no more than $10 \%$; the second level is of $5 \leq C \leq 8.1 \leq \mathrm{ctrl}<2.25 \leq D$ $<50,1.2 \leq R A<1.7$, occupying about $30 \%-45 \%$; the third grade is of $1 \leq C \leq 4.0 \leq \mathrm{ctrl}<1.0 \leq D<25.0 \leq R A$ $<1.2$ constituting more than $45 \%$, even $55 \%$ or more. The connectivity level of road network is statistically in pyramid-like structure (Table 1), which means that traffic axial lines that have absolute control over the whole network is relatively in minority and the intercity topological connectivity is relatively low. Consequently, the road network of Wuhan Metropolitan Area is not perfect enough as the network structure is comparatively fragile and its capability to deal with the unexpected network congestions is weak, which does do harm to the integration of metropolitan areas.

\subsection{The Spatial Morphology of Topological Network Is in Hub-and-Spoke and Fish Bone-Like Structure}

On the one hand, the entire metropolitan area network is mainly consisted of cities. Each central city like Wuhan, Jinzhou, Jinmen, Xinyang, Jiujiang, Suizhou etc. has become the crossing of traffic axial lines serving as the hub of the entire road network, while each intercity traffic axis serves as the spoke of the cross-linked road network, which all together make the whole road network the spatial structure of "hub-and-spoke" extending from the urban center to its circumference. On the other hand, some transport axis with higher connectivity and stronger control capability (No. 106 Backbone National Highway, No. 107 Backbone National Highway, 318 Backbone National Highway, Shanghai-Chengdu Expressway and Beijing-Zhuhai Expressway and other highways) constitute the entire road network space integrated core (just like series of backbone). The connectivity of those lower level transport axial lines (just like fishbone) connected to them aside is gradually lowered, thus the entire road network being in fish bone-like spatial morphology. The high-grade road network is at the early stage of its development, expected to be upgraded to a more mature network in network-like or cellular-like structure; The fish bone-like road network presents classified spatial extending sequence which indicates that the main transport axial lines in "fish bone" shape is of critical control significance; hence the most important transport axis in

Table 1. System of road network with hierarchy.

\begin{tabular}{cccc}
\hline Grades & Firstly & Secondly & Thirdly \\
Variables & 5.75 & 37.41 & 56.83 \\
Connectivity & 14.38 & 30.93 & 54.67 \\
Control value & 7.91 & 44.60 & 47.48 \\
Three-step depth value & 7.19 & 44.68 & 48.13 \\
Integration level & & & \\
\hline
\end{tabular}

the road network of Wuhan Metropolitan Area, whose technical parameters and road conditions directly affect the effectiveness and accessibility of the entire network. It constitutes the first level traffic axis. And those traffic axial lines which topologically speaking directly connected to the first level constitute the second level, which are mainly the provincial courses of better accessibility. This well reflects that the important transport axial lines can affect its circumference and at the same time expose penetration effect on it. Other provincial courses with lower connectivity and those municipal roads connected to the second level constitute the third level.

\subsection{The Highly Integrated Axial Lines Are Tensely Distributed along the City Corridors Crosswise, Constituting the Integrated Core of the Whole Region}

The integration reflects the degree of centralization or dispersion between a given axial-line and any other spatial units in an urban system, which reflects its permeability and core advantage. The highly integrated transport axial lines are mainly the radical channel crosslinked with Wuhan as its centre. The East-to-West band is from Jinzhou to Xiantao to Wuhan to E'zhou to Huangshi and the North-to-South from Xinyang to Xiaogan to Wuhan to Xianning to Yueyang, of which the integration values all exceed 1.70, making themselves the first level traffic axial lines that play the role as the integrative core of the whole metropolitan area with the strongest permeability and integration. Especially the crossing of the cross-like bands, Wuhan have become the network centre of the entire metropolitan area. All well highlights that the spatial morphology tendency of Wuhan Metropolitan Area is radically extended crosswise with Wuhan as its core (Figure 3).

\subsection{The Scale Distribution of Road Network Topological Connectivity Is Closely Related to the Cities' and Towns' System in the Metropolitan Area; and It Is the Same with the Transport Infrastructure and the Social and Economic Development}

Maintain high consistencies are the cities' and towns' scale distribution in the whole region, the spatial structure of the high level road network and its inter-city's social and economic development as well as the classified spatial differentiation of its traffic axial lines' connectivity and accessibility. Mainly depend on high-grade road network like freeways are traffic axial lines with better connectivity, higher control value, three-step depth value and integration level which often spatially extend along the node cities in larger scale where their intercity's social and economic exchanges are more frequently and are more significantly interacted. 

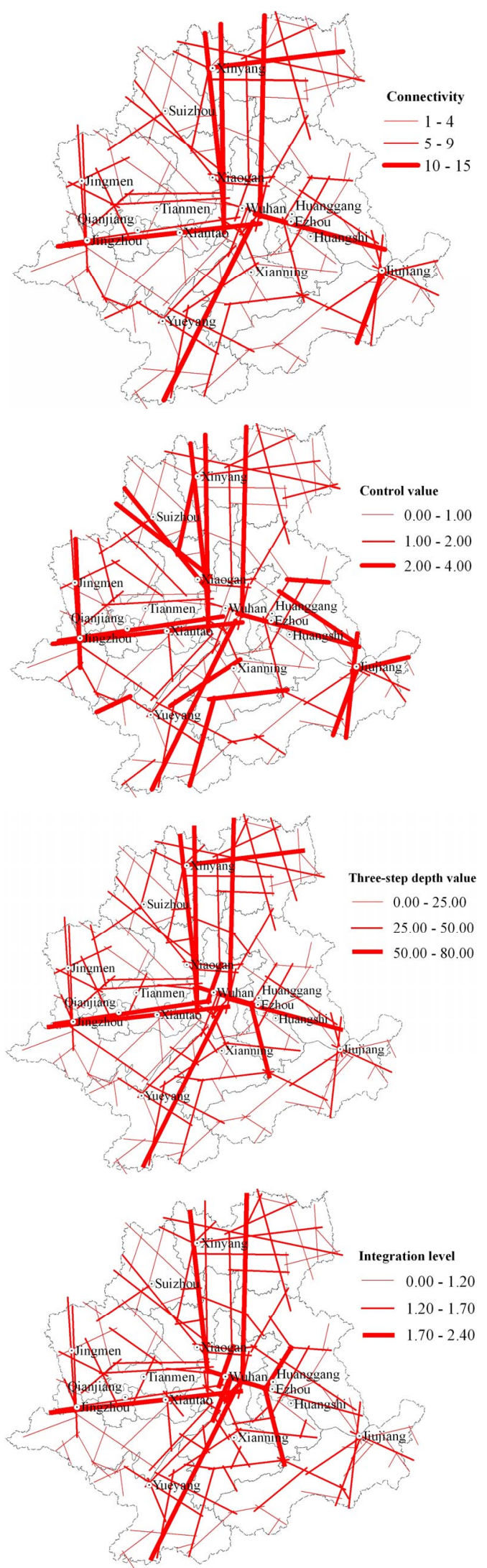

Figure 3. The spatial differentiation of road network in $\mathrm{Wu}$ han Metropolitan Area based on spatial syntactic.

\section{Conclusions and Recommendations}

\subsection{Conclusions}

- Axial network is in hub-and-spoke type and fish bonelike composite structure

The transport axial network is radically in hub-andspoke spatial structure with the urban center as its core and in fish bone-like structure with the highly integrated traffic axial lines as its framework. Maintain a high consistence are the topological connectivity level, the urban scale system and the regional social and economic development level as well as the transport infrastructure's current situation.

- The mean level of road network topological connectivity is relatively low; the gap between each level is noticeable; hence the inefficiency in its network servo.

The road network system leaves much to be desired with obvious gap and is of the pyramid-like shape. As the well-connected traffic axial lines are comparably inadequate and are relatively densely distributed, the network structure is fragile, thus resulting in its weaker capability to deal with the emergent network congestions and the lower efficiency of the whole network, which stands as an obstacle to its further stable extension and expansion. Meanwhile, the development level of the entire network is relatively low as there still are many "blind areas" in the high-grade road network, which does weaken the temporary road network servo capability. Consequently, there comes a good chance that the whole network can potentially be well extended and expanded.

- The gradation and classification of the road network cause the stratification of the accessible spatial morphology; hence the two ribbon-like Urban Corridor.

Due to the road network speed limits and the grade differentiation in its operational capacity, the servo quality and its capacity enjoyed by the nodes in the network is significantly differentiated. The network accessible space presents spatial differentiation. The node cities along the Beijing-Zhuhai and Shanghai-Chengdu Freeway become the best domain in the whole network in terms of its connectivity and accessibility. Namely, there comes classification in the servo quality in the band-like network, whose hub-Wuhan taking advantage of its central location has become the distribution center in the whole metropolitan network. The crosswise radical traffic axial lines are expected to be developed into the main axis for the economic development and into the city corridors of the whole metropolitan area.

\subsection{Suggestions}

Strengthen the construction of transport infrastructure, especially those high level traffic axial lines and nodes that have greater influences on the regional accessibility; 
expand the road network coverage and network size; increase the total number of network loops; strengthen the servo capability and effective control ability of its high grade network over Wuhan metropolitan network. Particularly the vast area like Huanggang, Xinyang to the east of the whole region and Xianning to the south, their development level of the high grade road network expected to be further improved is relatively low, which limits the harmonious and even development of the whole region's development.

More obvious is the single polarization of the regional network. More prominent is Wuhan's control effect over the whole metropolitan road network. Therefore, cities like Jingzhou, Huangshi and Xiaogan should be moderately developed to serve as the secondary urban centers in the metropolitan road network. In this way, multipolarization situation can be formed, which is beneficial to the even development of the metropolitan area spatial accessibility morphology, thus increasing the balance and stability of the whole road network.

Main contact channels (high-grade traffic axial lines) are in minority. Therefore once the whole network encounters emergency, its self-adaptation and self-regulation capability becomes weak. This could easily bring about the bottleneck effect, and have negative impacts on the validity and reliability of the network, and even make the whole network crash down. As a result, we should strengthen the transformation and construction of those important traffic axial lines in the road network, speed up inter-cities' express railway and highway construction with Wuhan city as its center. In this way, a new batch of high-grade traffic axial lines will be built, which is beneficial to the achievement of the appropriate distribution of the road network and the improvement of the overall network operation level and its efficiency. In particular, we should strength the construction of the freeway network in the whole region, which is of great significance to the improvement of the regional accessibility and the common economic and social development of the whole region.

\section{Acknowledgements}

This paper was supported by National Social Science Foundation of China (Project ID: 11CJL048).

\section{REFERENCES}

[1] X. Cao, X. Yan, et al., "Research on Spatial Evolution in Guangzhou-Shenzhen-Hong Kong Mega-City Corridor," The Commercial Press, Beijing, 2006, p. 35.

[2] P. Li and Y. Lu, "Review and Prospection of Accessibility Research," Progress in Geography, Vol. 24, No. 3, 2005, pp. 69-78.

[3] W. G. Hansen, "How Accessibility Shapes Land-Use," Jour- nal of the American Institute of Planners, Vol. 25, No. 2, 1959, pp. 73-76. doi:10.1080/01944365908978307

[4] D. C. Hodge, "Accessibility-Related Issues", Journal of Transport Geography, Vol. 5, No. 1, 1997, pp. 33-34. doi:10.1016/S0966-6923(96)00050-6

[5] G. H. Pirie, "Measuring Accessibility: A Review and Proposal," Environment and Planning A, Vol. 11, No. 3, 1979, pp. 299-312. doi:10.1068/a110299

[6] J. Chen, F. Lu and C. Cheng, "Advance in Accessibility Evaluation Approaches and Applications," Progress in Geography, Vol. 26, No. 5, 2007, pp. 100-110.

[7] M. E. O'Kelly, “A Geographer's Analysis of Hub-andSpoke Networks," Journal of Transport Geography, Vol. 6, No. 3, 1998, pp. 171-186. doi:10.1016/S0966-6923(98)00010-6

[8] J. Wang and F. Jin, "Railway Network Organization and Spatial Service System Optimization in China," Acta Geographica Sinica, Vol. 60, No. 3, 2005, pp. 371-380.

[9] V. Roger, K. Spiekermann, et al., "Accessibility and Economic Development in Europe," Regional Studies, Vol. 33, No. 1, 1999, pp. 1-15. doi:10.1080/00343409950118878

[10] F. Bruinsma, "The Accessibility of European Cities," Environment and Planning A, Vol. 30, No. 3, 1998, pp. 499521. doi:10.1068/a300499

[11] J. Gutierrez, "Location, Economic Potential and Daily Accessibility: An Analysis of the Accessibility Impact of the High-Speed Line Madrid-Barcelona-French Border," Journal of Transport Geography, Vol. 9, No. 4, 2001, pp. 229-242. doi:10.1016/S0966-6923(01)00017-5

[12] S. Li and Y. Shum, "Impacts of the National Trunk Highway System on Accessibility in China," Journal of Transport Geography, Vol. 9, No. 1, 2001, pp. 39-48. doi:10.1016/S0966-6923(00)00040-5

[13] F. B. David, "Network Cities: Creative Urban Agglomerations for the 21st Century," Urban Studies, Vol. 32, No. 2, 1995, pp. 313-327. doi:10.1080/00420989550013103

[14] L. Bertolini, "Mobility Environment and Network Cities," Journal of Urban Design, Vol. 8, No. 1, 2003, pp. 27-43. doi:10.1080/1357480032000064755

[15] F. Jin and J. Wang, "Railway Network Expansion and Spatial Accessibility Analysis in China: 1906-2000," Acta Geographica Sinica, Vol. 59, No. 2, 2004, pp. 293-302.

[16] X. Cao and X. Yan, "The Impact of the Evolution of Land Network on Spatial Structure of Accessibility in the Developed Areas: The Case of Dongguan City in Guangdong Province," Geographical Research, Vol. 22, No. 3 , 2003, pp. 305-313.

[17] D. E. F. Voskuhl, "Interlinking the Region with Its Centre: The Example of the Karlsruhe in Germany," Journal of Transport Geography, Vol. 3, No. 4, 1995, pp. 281-286. doi:10.1016/0966-6923(95)00026-7

[18] W. A. Muraco, "Intra-Urban Accessibility," Economic Geography, Vol. 48, No. 4, 1972, pp. 388-405. doi: $10.2307 / 142890$

[19] D. O'Sullivan, A. Morrison and J. Shearer, "Using Desk- 
top GIS for the Investigation of Accessibility by Public Trans- port: An Isochrones Approach," International Journal of Geographical Information Science, Vol. 14, No. 1, 2000, pp. 85-104. doi:10.1080/136588100240976

[20] C. Cheng, W. Zhang, J. Chen, et al., "Evaluating the Accessibility about Beijing's Subways in 2008 Based on Spatial Syntax," Geo-Information Science, Vol. 9, No. 6, 2007, pp. 31-35.

[21] M. Chen, F. Shen, L. Zha, et al., "A Research on Urban Traffic Network Based on Space Syntax: A Case Study on Wuhu City," Geography and Geo-Information Science, Vol. 21, No. 2, 2005, pp. 39-42.

[22] W. Wu, Y. Cao and W. Cao, "Spatial Structure and Evolution of Highway Accessibility in the Yangtze River Delta," Acta Geographica Sinica, Vol. 61, No. 10, 2006, pp. 1065-1074.

[23] J. Bowen, "Airline Hubs in Southeast Asia: National Economic Development and Nodal Accessibility," Journal of Transport Geography, Vol. 8, No. 1, 2000, pp. 25-41. doi:10.1016/S0966-6923(99)00030-7

[24] S. Fatal, "Land Re-Organization in Relation to Make in an Indian City," Land Use Policy, Vol. 18, No. 2, 2001, pp. 191-199. doi:10.1016/S0264-8377(01)00007-2
[25] T. Dai, F. Jin and J. Wang, "Spatial Interaction and Network Structure Evolvement of Cities in Term of China's Railway Passenger Flow in 1990s," Progress in Geography, Vol. 24, No. 2, 2005, pp.80-89.

[26] J. Li, "A Study of Comparisons of Urban Competitiveness and Accessibility in Shanxi Province," Geographic Science, Vol. 28, No. 4, 2008, pp. 471-477.

[27] L. Zhang and Y. Lu, "Regional Accessibility of Land Traffic Network in the Yangtze River Delta," Journal of Geographical Sciences, Vol. 17, No. 3, 2007, pp. 351-364. doi:10.1007/s11442-007-0351-z

[28] C. Liu, M. Ding, Z. Zhang, et al., "Accessibility Analysis on the Spatial Linkage of Wuhan Metropolitan Area," Progress in Geography, Vol. 26, No .6, 2007, pp. 96-108.

[29] B. Hillier, "Configurational Modeling of Urban Movement Networks," Environment and Planning B: Planning and Design, Vol. 25, No. 1, 1998, pp. 59-84.

[30] C. Gu, "Urban Geography of China," The Commercial Press, Beijing, 1999, p.164.

[31] C. Liu, R. Yu, J. Xiong, et al., "The Spatial Structure of Economic Linkage of Wuhan Metropolitan Area", Geographical Research, Vol. 26, No. 1, 2007, pp. 197-209. 